\title{
Can a unisegmental pulmonary infarct be lethal?
}

\author{
Abdelmoughit Hosni ${ }^{1}$, Siham EL HADDAD ${ }^{1}$, Nazik ALLALI ${ }^{1}$, and Latifa CHAT ${ }^{1}$ \\ ${ }^{1}$ Children's hospital in Rabat
}

December 15, 2021

\begin{abstract}
To highlight the risk of septic complications following a pulmonary embolism (PE), we report the case of an elderly patient, that was hospitalized for a PE, an had progressively worsen his respiratory and septic state. Chest CT slices showed a gangrenous lung segment with an associated pleuropulmonary abscess.
\end{abstract}

TITLE: Can a unisegmental pulmonary infarct be lethal?

AUTHORS: Abdelmoughit HOSNI, Siham EL HADDAD, Nazik ALLALI, Latifa CHAT

AFFILIATION: Department of pediatric radiology, Children's hospital in Rabat, Rabat, Morocco

Correspondant author:Abdelmoughit HOSNI

Email: abdelmoughith@gmail.com

KEYWORDS: Pulmonary infarct - Pulmonary embolism - Sepsis - Abscess - Complications

Written informed consent was obtained from the patient to publish this report in accordance with the journal's patient consent policy

\section{TEXT:}

A 78 years-old patient, admitted to our cardiology clinic for a pulmonary embolism involving the right lower and middle lobar arteries. At admission, he was stable and benefited from an anticoagulative therapy based on LMWH. The evolution was marked by a progressive dyspnea and cough. Then, serial chest radiographs were realized, showing an increasing right pleural effusion. At day 12 of his admission, he installed a tachycardia at $104 \mathrm{ppm}$, with a high inflammatory blood markers (WBC:22000/mm3, CRP: 305 $\mathrm{mg} / \mathrm{L}$, Procalcitonine: $6 \mathrm{ng} / \mathrm{mL}$ ). A contrast-enhanced chest CT was managed (Figure) showing a large right pleuropulmonary collection, including a fluid-air level, with a visceral pleural enhancement. The lateral segment of the middle lobe was collapsed and gangrenous. A percutaneous pleural drainage was performed, with identification of Klebsiella pneumoniae in the liquid culture. He benefited from a large specter antibiotic and was discharged after 4 more weeks of hospitalization.

Pulmonary infarcts don't systematically occur in pulmonary embolism, due to the dual nature of pulmonary blood flow.[1,2] However, they are a well-recognized cause of cavitation, mostly in elderly patients with comorbid conditions. [2] Herein, cavitation is faster with infected pulmonary infarcts than with bland aseptic ones. [1,2] Then, complications such as pneumothorax, empyema, and bronchopleural fistula may occur leading to lethal conditions. [1]

CONFLICT OF INTEREST: None

FUNDING SOURCE: None

ETHICAL APPROVAL STATEMENT: Not required 


\section{REFERENCES:}

Koroscil MT, Hauser TR. Acute pulmonary embolism leading to cavitation and large pulmonary abscess: A rare complication of pulmonary infarction.Respir Med Case Rep . 2016;20:72-74. Published 2016 Dec 18. doi:10.1016/j.rmcr.2016.12.001

Rajagopala S, Devaraj U, D’Souza G. Infected cavitating pulmonary infarction. Respir Care . 2011;56(5):707709. doi: $10.4187 /$ respcare.00828

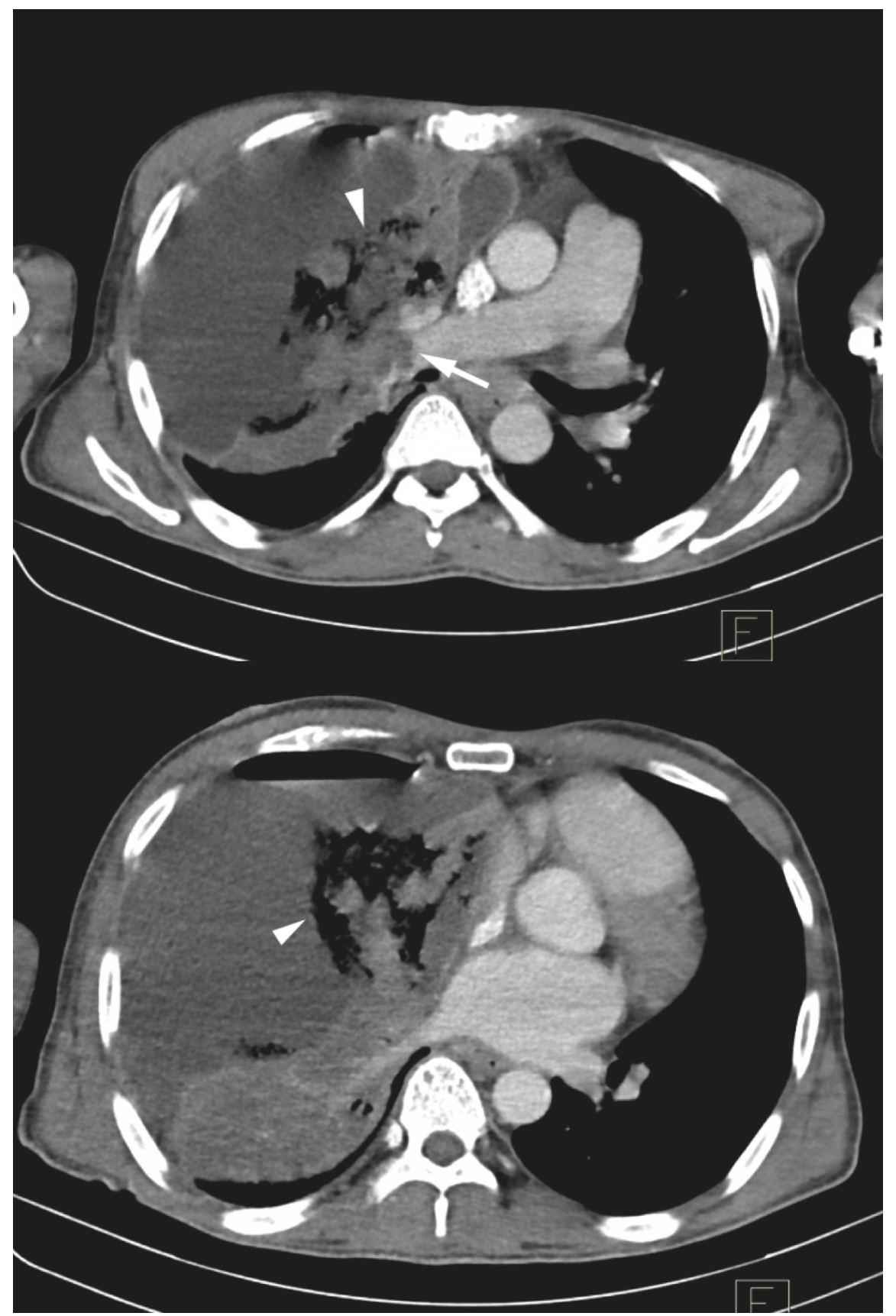

